REVESCO. Revista de Estudios Cooperativos

ISSN: $1885-8031$

http://dx.doi.org/10.5209/REVE.58394

\title{
Crisis económica y economía social
}

\author{
Manuel Jaén García ${ }^{1}$
}

Recibido: 30 de septiembre de 2017 / Aceptado: 21 de noviembre de 2017

Resumen. La crisis económica de 2008 golpeó fuertemente los diversos sectores de la economía española. Aunque el punto de partida fue la explosión de la burbuja inmobiliaria seguida de la caída de gran parte de las antiguas cajas de ahorro, se extendió a toda la economía dejando tras de sí un reguero de cierres empresariales y paro masivo así como un sector público que, tras rescatar las cajas y bancos en crisis, se encontró con un enorme agujero en las cuentas públicas y un diferencial en los pagos de intereses de la deuda (la denominada prima de riesgo) con respecto a la deuda alemana que llegó a superar los 500 puntos básicos. La intervención del Banco Central Europeo (BCE) con su compra masiva de deuda soberana permitió, en parte, superar esta situación propiciando una economía en crecimiento en los últimos años (2014 en adelante). En este contexto se sitúa el presente trabajo en el que se estudia el comportamiento de las empresas de economía social durante el período de crisis en comparación con la economía en general. Para ello se consideran los indicadores más usuales y se comparan las dos vertientes: el sector empresarial en general y el sector de la economía social en particular.

Palabras clave: Economía social; Crisis económica; Valor añadido bruto; Beneficios; Empleo. Claves Econlit: A10; A13; E32.

\section{[en] Economic crisis and social economy}

\begin{abstract}
The economic recession of 2008 deeply impacted numerous sectors in the Spanish economy. Although this crisis was sparked by the housing bubble followed by the demise of a large part of now defunct savings banks, it spread throughout the entire economy leaving a trail of business closures and massive unemployment. In addition, after having bailed out the banks during the recession, the public sector was left with an enormous hole in public finances and an interest rate differential on debt payments (the so-called risk premium) that surpassed 500 basis points with respect to German debt. Intervention by the European Central Bank (ECB), which consisted of a massive purchase of sovereign debt, allowed the country, in part, to overcome this situation and foster a growing economy in recent years (2014 onwards). The present work is set within this context and studies the behavior of social enterprises during the crisis period in comparison with the economy in general. With this aim, the most common indicators are utilized and the two sectors are compared: the general business sector and the social enterprise sector in particular.
\end{abstract}

Keywords: Social economics; Economic crisis; Gross added value; Benefits; Employment.

1 Universidad de Almería, España

Dirección de correo electrónico: mjaen@ual.es 
Sumario. 1. Introducción. 2. Aspectos generales de la economía social. 3. La crisis económica y sus consecuencias. 4. La empresa de economía social en el marco económico actual. 5. Conclusiones. 6. Referencias bibliográficas.

Cómo citar: Jaén García, M. (2017) Crisis económica y economía social. REVESCO. Revista de Estudios Cooperativos, Monográfico, № 126, pp. 74-93. DOI: 10.5209/REVE.58394.

\section{Introducción}

Este trabajo realiza un sencillo análisis comparativo de la reacción frente a la crisis económica de las empresas de economía social en relación al sector empresarial en general.

La hipótesis inicial es que, dadas sus características, las empresas de economía social deben haber reaccionado con mayor fortaleza en aquellas partes que han sido más vulnerables a la crisis. Es decir, en cuanto a la pérdida de empleo y la disminución de los salarios. Se espera un comportamiento análogo en cuanto a creación o destrucción de empresas o beneficios empresariales dado que la distinción de las empresas de economía social con respecto al resto de empresas es en cuanto a su estructura organizativa interna en la que algunos o todos los empleados son socios de las empresas y no en cuanto al objeto social que puede ser cualquiera de los posibles en una economía de mercado.

En la siguiente sección se enmarca el trabajo para lo que se consideran las definiciones de economía social y, en particular, las de cooperativa y sociedad anónima laboral. En la tercera se analiza la evolución de la economía española en los años precedentes y durante la crisis económica. En la cuarta se comparan, mediante la utilización de gráficos, diferentes indicadores de las empresas de economía social con los correspondientes de las empresas de la economía española en general. En la quinta se resume el trabajo y se elaboran las conclusiones del mismo.

\section{Aspectos generales de la economía social}

La ley 5/2011 de economía social la define como "conjunto de actividades económicas y empresariales, que en el ámbito privado llevan a cabo aquellas entidades que, de conformidad con los principios recogidos en el artículo 4, persiguen bien el interés colectivo de sus integrantes, bien el interés general económico o social, o ambos".

Por su parte los principios recogidos en el artículo 4 son los siguientes:

a) Primacía de las personas y del fin social sobre el capital, que se concreta en gestión autónoma y transparente, democrática y participativa, que lleva a priorizar la toma de decisiones más en función de las personas y sus aportaciones de trabajo y servicios prestados a la entidad o en función del fin social, que en relación a sus aportaciones al capital social.

b) Aplicación de los resultados obtenidos de la actividad económica principalmente en función del trabajo aportado y servicio o actividad 
realizada por las socias y socios o por sus miembros y, en su caso, al fin social objeto de la entidad.

c) Promoción de la solidaridad interna y con la sociedad que favorezca el compromiso con el desarrollo local, la igualdad de oportunidades entre hombres y mujeres, la cohesión social, la inserción de personas en riesgo de exclusión social, la generación de empleo estable y de calidad, la conciliación de la vida personal, familiar y laboral y la sostenibilidad.

d) Independencia respecto a los poderes públicos.

El objetivo, según el artículo 1, es "...establecer un marco jurídico común para el conjunto de entidades que integran la economía social, con pleno respeto a la normativa específica aplicable a cada una de ellas, así como determinar las medidas de fomento a favor de las mismas en consideración a los fines y principios que les son propios".

La ley considera entidades de la economía social a las cooperativas, las mutualidades, las fundaciones y las asociaciones que lleven a cabo actividad económica, las sociedades laborales, las empresas de inserción, los centros especiales de empleo, las cofradías de pescadores, las sociedades agrarias de transformación y las entidades singulares creadas por normas específicas que se rijan por los principios establecidos en el artículo anterior. "Asimismo, podrán formar parte de la economía social aquellas entidades que realicen actividad económica y empresarial, cuyas reglas de funcionamiento respondan a los principios enumerados en el artículo 4, y que sean incluidas en el catálogo de entidades establecido en el artículo 6 de esta Ley". ${ }^{2}$

De acuerdo con Algora (2011) las empresas de economía social poseen unos potenciales y adquieren unos compromisos que les otorgan un valor añadido que vale la pena destacar:

- $\quad$ En primer lugar, la creación de puestos de trabajo de mayor estabilidad y calidad y la flexibilidad interna para el mantenimiento de los mismos.

- En segundo lugar, la solidaridad y la vinculación con el entorno, fomentando el desarrollo local y la no deslocalización.

- Y por último la inclusión sociolaboral de personas con discapacidad y/o en riego de exclusión.

Dados los datos de que disponemos consideramos del total de la economía social a cooperativas y sociedades laborales y dentro de estas últimas a las sociedades anónimas y limitadas.

Las cooperativas se definen por la Alianza Cooperativa Internacional (1995: 17) como "asociaciones anónimas de personas que se unen de forma voluntaria para satisfacer sus necesidades y aspiraciones económicas, sociales y culturales en común mediante una empresa de propiedad conjunta y de gestión democrática".

Se consideran dos tipos de valores en las cooperativas, valores básicos tales como ayuda mutua, autorresponsabilidad, democracia, igualdad, equidad y

2 En Bacchiega y Borgaza (2003) definición de tercer sector. En Chaves y Monzón (2008) definición de economía social. 
solidaridad y valores éticos tales como honestidad, transparencia, responsabilidad social y preocupación por los demás.

Es responsabilidad de la cooperativa no sólo la obtención de un beneficio económico sino también la mejora de la calidad de vida y de la situación económica de los socios, así como de la comunidad geográfica dónde desarrolla su actividad.

Las sociedades laborales se definen como organizaciones híbridas entre las empresas capitalistas y las empresas de participación (cooperativas) ya que al menos un $50 \%$ del capital ha de estar en manos de socios trabajadores vinculados a la empresa con un contrato laboral indefinido y poseedores de acciones de clase laboral. Por otra parte, pueden admitir socios capitalistas que mantienen acciones de carácter general y que únicamente persiguen el lucro individual. Buscando una dilución del capital, el máximo de acciones del socio trabajador es del $33 \%$ del capital social. La estructura organizativa y la composición del accionariado es similar al de las cooperativas de trabajo asociado.

Las investigaciones empíricas realizadas muestran que, en gran parte, los socios capitalistas son familiares de los socios trabajadores (Sáez y González, 2005).

Las sociedades laborales permiten la puesta en marcha de proyectos colectivos dónde los trabajadores son los propios gestores de la actividad empresarial. Desde el punto de vista de la creación de empleo, la principal ventaja frente a otro tipo de empresa es su mayor flexibilidad lo que la hace más permeable a las situaciones de crisis. En épocas de recesión, los gestores de la sociedad antes de disolverla aceptarán un menor nivel de actividad y menores retribuciones para los socios trabajadores, motivo por el que cabe esperar una mayor estabilidad en el empleo (Pérez y otros, 2008; Algora, 2011; Román, 2014; Sala y otros, 2014; Bretos y Morandeira, 2016).

En los siguientes apartados se estudia cómo ha incidido la crisis sobre las empresas de economía social y qué se debe esperar de estos modelos empresariales a la hora de afrontar el contexto económico actual.

\section{La crisis económica y sus consecuencias}

La crisis económica de 2008, denominada la Gran Recesión, tuvo un fuerte impacto en las economías de Estados Unidos, Europa y España. La caída en la producción española fue acompañada por caídas en el empleo, salarios y beneficios empresariales y no fue hasta 2015, cuando la economía empezó a tener resultados positivos, tal como se observa en el gráfico 1 que muestra un crecimiento positivo del PIB hasta 2008, aunque con tasas de crecimiento muy bajas, una pequeña recuperación en 2013 con caída de nuevo en 2014 y otra pequeña recuperación en 2015. 
Gráfico. 1.
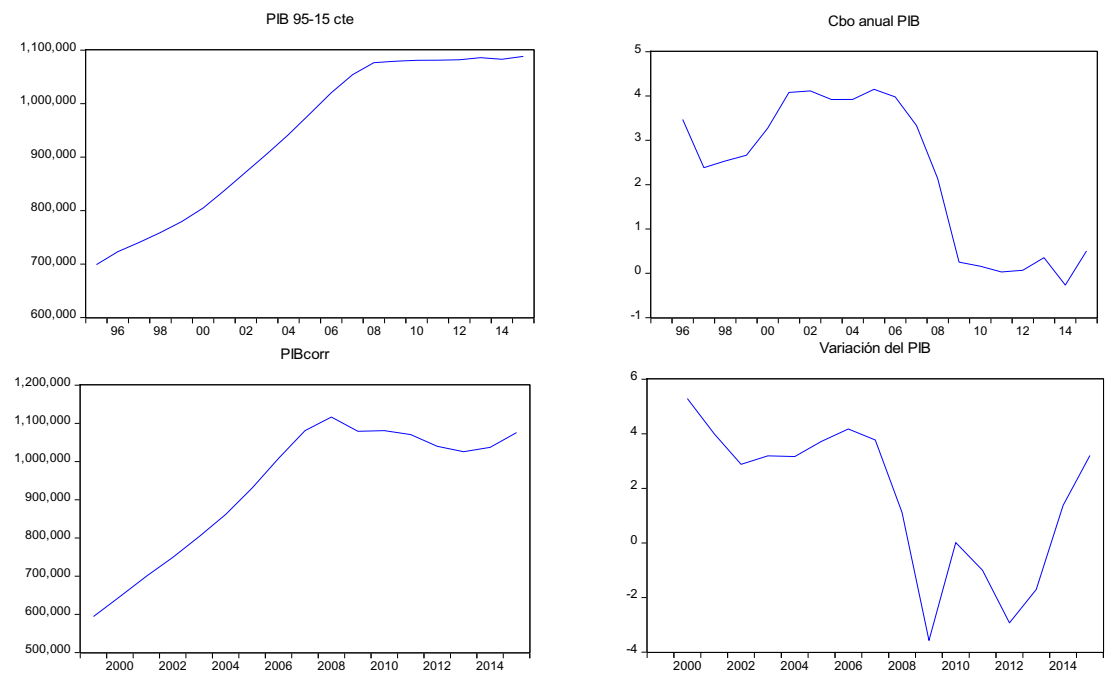

Desde 1992 la economía española tuvo un fuerte crecimiento que continuó su senda positiva hasta el momento de la crisis de 2008. Este crecimiento vino determinado por diversos factores. En primer lugar, la entrada de España en la Unión Europea (UE) trajo consigo una fuerte bajada en los tipos de interés y las ayudas que recibimos propiciaron una rápida construcción de infraestructuras tales como autopistas y aeropuertos. En 1992 los acontecimientos simultáneos de la Expo 92 y las olimpiadas de Barcelona permitieron la creación de empleo masivo en los sectores de la construcción y los servicios y un clima masivo de optimismo. Por otra parte, ante las ventajas de los menores salarios en España con respecto a otros países europeos, muchas industrias europeas y de otros países se instalaron en nuestro suelo. La liberalización del suelo en 1998 trajo consigo un enorme aumento de la construcción tanto en zonas turísticas como del interior promoviendo la compra de viviendas por parte del sector bancario con una gran liquidez y con la posibilidad de acudir a los mercados exteriores. Al mismo tiempo se produjo la llegada masiva de emigrantes al hilo de la fuerte creación de empleo que se estaba produciendo en esos momentos, sobre todo en el sector de la construcción y en los servicios (sector turístico).

El PIB tuvo un crecimiento continuado en el período con máximos del $4,1 \%$ con respecto al año anterior en 2001 y 2002 y una media entre 1995 y 2008 del $3,38 \%$. Al mismo tiempo se creó empleo de forma masiva pasándose de una tasa de paro del 20,3\% en 1992 al 8,6\% en 2007. Aunque ésta es una tasa de paro elevado en términos de otros países europeos y Estados Unidos es un valor muy bajo en relación a los valores pasados en España.

Entre 1994 y 1998 se produjo un drástico cambio en el sector público español. Conceptualmente fue una respuesta al cambio de paradigma económico desde el modelo social-demócrata postkeynesiano al sistema liberal-neoclásico. Las razones básicas para este cambio se encuentran en la necesidad de cumplir en su totalidad las condiciones del tratado de Maastrich así como el pacto de estabilidad y 
crecimiento (PEC), en conjunción con que este período coincide con el principio de la fase ascendente del ciclo económico en 1996. Los elevados déficits presupuestarios (7,3\% del PIB en 1994) caen al 2,6\% en 1998, cumpliendo de forma estricta las condiciones del PEC (3\%) con respecto a los déficits presupuestarios.

Entre 1999 y 2007 se produce la consolidación de las finanzas públicas en España debido al PEC para propiciar la entrada de España en la Unión Económica y Monetaria (UE, 2002) que empujó al sector público al cumplimiento de las condiciones de Maastrich (3\% del PIB de déficit público en las cuentas anuales y $60 \%$ del PIB para el total de la deuda pública) y a la expansión del sector inmobiliario que hizo posible un crecimiento sin precedentes acompañado de un aumento de la recaudación fiscal en los diferentes impuestos. De esta forma en 2001 se logra un presupuesto equilibrado, aunque el gasto público supone el $40 \%$ del PIB, lo que supone una subida del $4 \%$ con respecto al promedio del periodo 1997-2001.

El período de la burbuja posibilitó un fuerte crecimiento del empleo unido a un gran aumento de la venta de viviendas lo que dio lugar a un aumento de la recaudación fiscal tanto directa, vía IRPF por el incremento del empleo tanto en el sector de la construcción como en otros sectores, e indirecta debido a la recaudación por IVA de la venta de vivienda nueva y el impuesto de transmisiones patrimoniales en la venta de vivienda usada. Por otra parte aumentó la recaudación en impuestos locales derivados de la construcción de viviendas.

De 2007 a 2014 se produce una fuerte caída en la economía española debido al pinchazo de la burbuja inmobiliaria. Inicialmente, la expansión continuó y el PIB español alcanzó el promedio de la UE 27. En ese momento, la economía española arrastrada por la caída en las economías estadounidenses y europeas y sus propios problemas internos, entró en una profunda recesión de la que empezó a emerger en 2014 con crecimiento positivo aunque modesto $(1,2 \%)$ del PIB y con déficits presupuestarios del 7\% en 2013 y 6\% en 2014.

\section{La empresa de economía social en el marco económico actual}

El propósito de este apartado es comparar la reacción de la economía española en general y de la economía social (cooperativas y sociedades laborales) a la crisis económica, considerando las repercusiones en el VAB, empleo, beneficios empresariales, salarios y demás variables que definen a las empresas. Los datos están tomados de la página web del Instituto Nacional de Estadística (INE) para la economía en general y de la Dirección General de la Economía Social, del Trabajo Autónomo y de la Responsabilidad Social de las Empresas del Ministerio de Empleo y Seguridad Social ${ }^{3}$. En estos últimos datos no se incluyen País Vasco y Navarra.

En el gráfico 2 se considera el VAB tanto de la economía general como de la social, su evolución y el porcentaje que supone el VAB social en relación al general. El VAB mide el valor añadido generado por el conjunto de productores de

Los datos disponibles abarcan el período entre 1999 y 2014 
un área económica, recogiendo en definitiva los valores que se agregan a los bienes y servicios en las distintas etapas del proceso productivo. Por tanto es un buen indicador de la aportación a la economía de diferentes sectores y permite analizar la evolución de la economía mediante su medición.

Gráfico. 2.

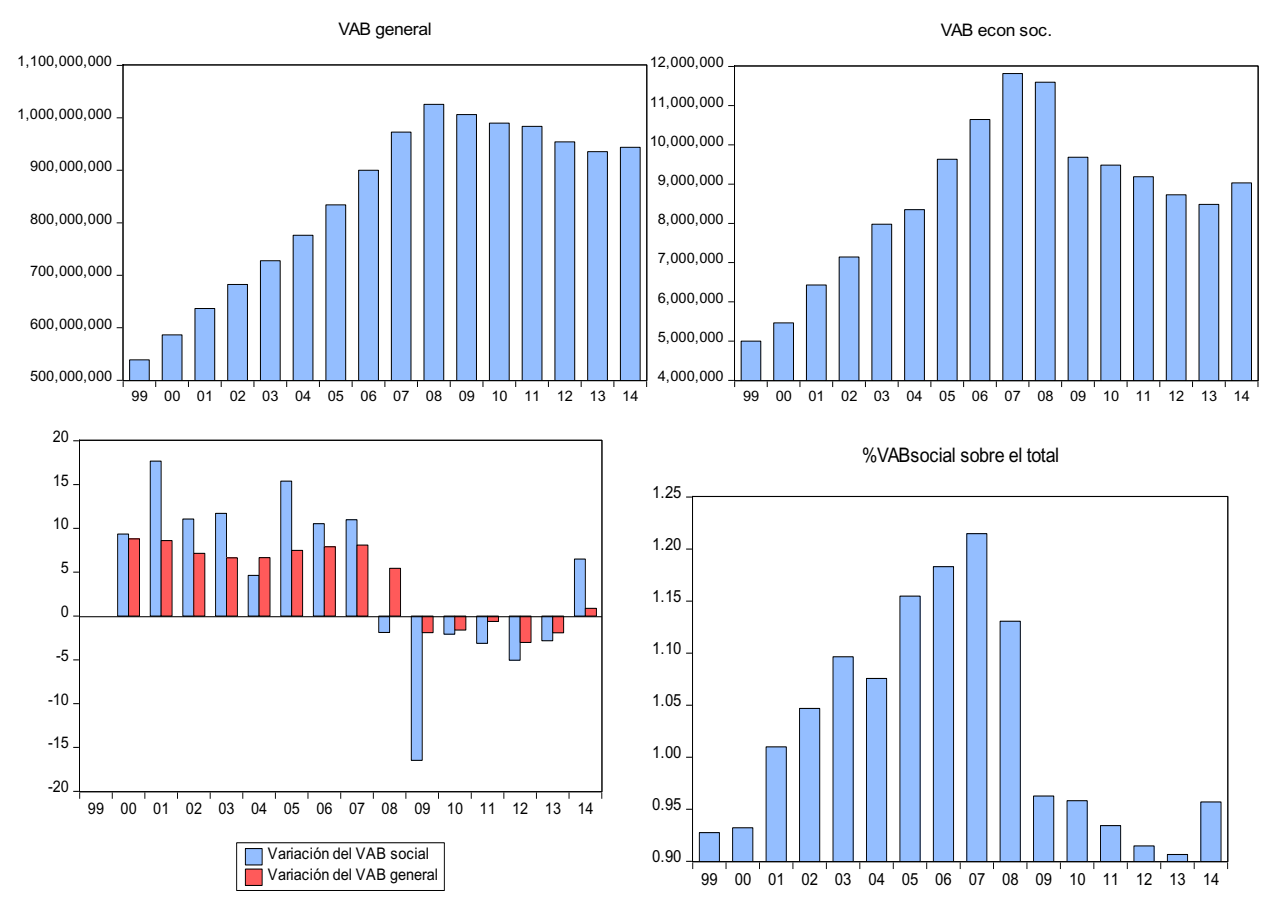

Se observa como el VAB de la economía en general sigue una senda de crecimiento con un máximo en 2008 y un suave decrecimiento a partir de ese año que concluye en 2013 aunque sin volver a alcanzar el valor de 2008. Por su parte el VAB correspondiente a la parte de la economía social que estamos considerando sigue una senda de crecimiento hasta 2007 descendiendo bruscamente a partir de 2009 hasta 2013 creciendo a partir de 2014. Por otra parte se observa que el porcentaje del VAB social sobre el VAB total alcanzó su máximo en 2007 con un valor del 1,21\% descendiendo posteriormente hasta alcanzar su mínimo en 2013 con un $0,906 \%$ y volver a crecer en 2014 con un $0,956 \%{ }^{4}$. A simple vista se puede deducir que el sector de la economía social muestra un comportamiento procíclico y que su recuperación en 2014 es más rápida que la de la economía en general, aunque no se pueden hacer afirmaciones rotundas dada la limitación de datos.

En el gráfico 3 se considera la evolución del número de empresas en general y del número de empresas de la economía social. Dado que los datos para la economía social sólo están disponibles entre 1999 y 2014, sólo se dispone de 15

4 Como anexo a este trabajo se incluyen diferentes cuadros explicativos. 
observaciones para cada una de ellas. No obstante, se puede ver la evolución que se ha producido en los últimos años. El número de empresas aumenta hasta alcanzar un máximo en 2008 de 3.427.239 y, aunque a partir de ahí empieza a descender, el descenso es muy lento alcanzando el mínimo en 2013 con 3.146 .570 empresas. Es decir, el máximo de disminución fue del 8,76\%. Las empresas de economía social constituyen una parte muy pequeña del total de las empresas en la economía pues suponen el 1,4\% del total de empresas. El número máximo de este tipo de empresas se alcanza en 2006 con 45.821 y a partir de ahí ha ido decreciendo hasta situarse en 31.085 en 2014 , lo que supone un descenso del $31,3 \%$. Las razones hay que buscarlas en la fragilidad de este tipo de empresas en cuanto a posibilidades de resistir una situación crítica pues su capital suele ser muy pequeño y tienen poco acceso a canales de financiación y cuando lo tienen suele ser muy caro. Según datos de la propia dirección general sólo el 10,59\% de las empresas de economía social sobreviven más de 20 años y el 24,94\% más de 10 años, sucumbiendo en su primer año de vida el 13,5\% de dichas empresas. Esto es debido, en parte, al sector al que pertenecen estas empresas. Aunque no hay datos oficiales creemos, y los datos de variación de empleo lo confirman, que se destruyeron bastantes empresas en sectores vulnerables a la crisis tales como construcción y hostelería.

\section{Gráfico. 3.}
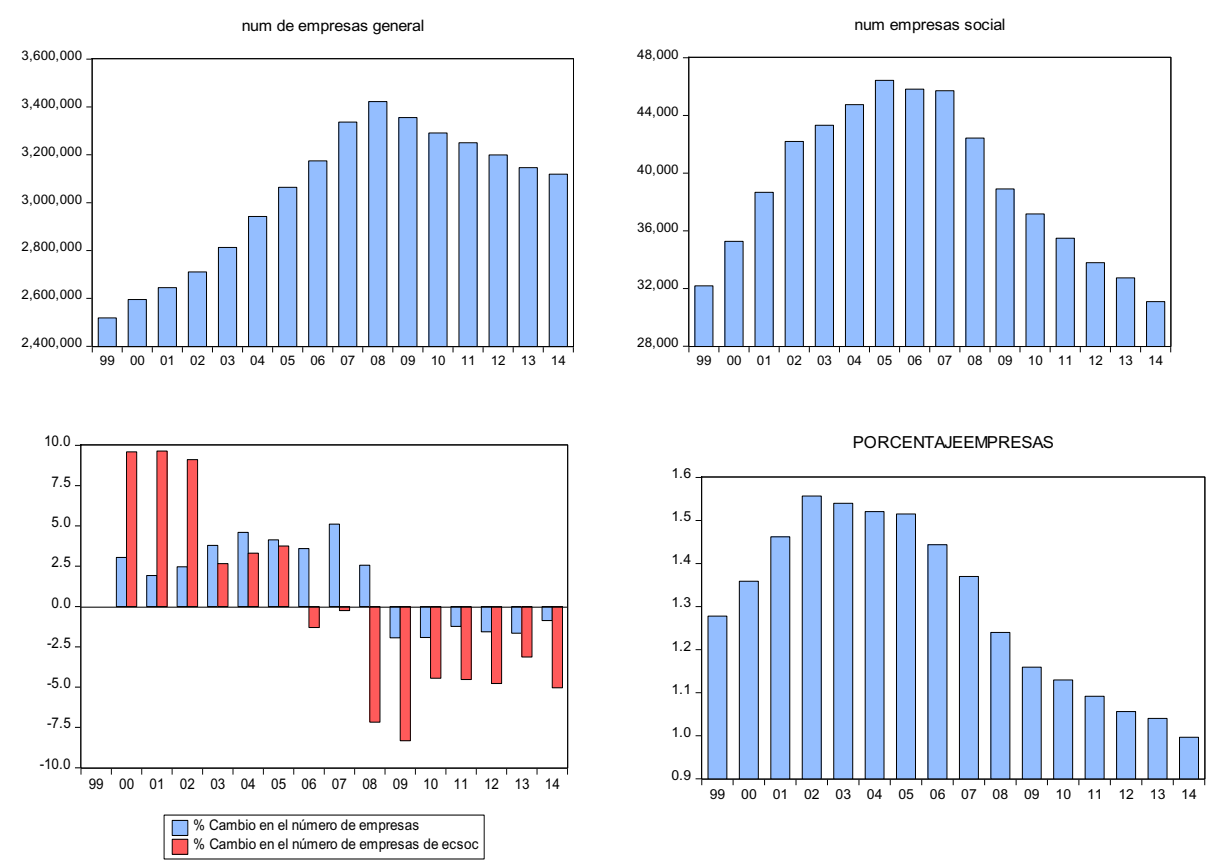

Por último, analizamos el porcentaje de empresas de la economía social en relación al total. El máximo se alcanzó el 2002 con un 1,55\% y a partir de ahí se ha producido una disminución hasta alcanzar únicamente el 0,99\% en 2014. 
De mayor interés es la comparación en la variación año a año del número de empresas. En la economía social los años entre 1999 y 2005 son de crecimiento muy fuerte en los primeros años y más moderado después. Es a partir de 2008 cuando se produce un descenso continuado y muy elevado en los dos primeros años, descenso que en 2014 no había revertido. Por su parte, las empresas de la economía en general tienen un aumento entre 1999 y 2007 que aunque lento es continuado. El descenso a partir de dicho año es asimismo bastante lento aunque en 2014 aún no se había producido una recuperación.

En el gráfico 4 se observa la evolución del número de trabajadores en ambos tipos de empresas.

\section{Gráfico. 4.}
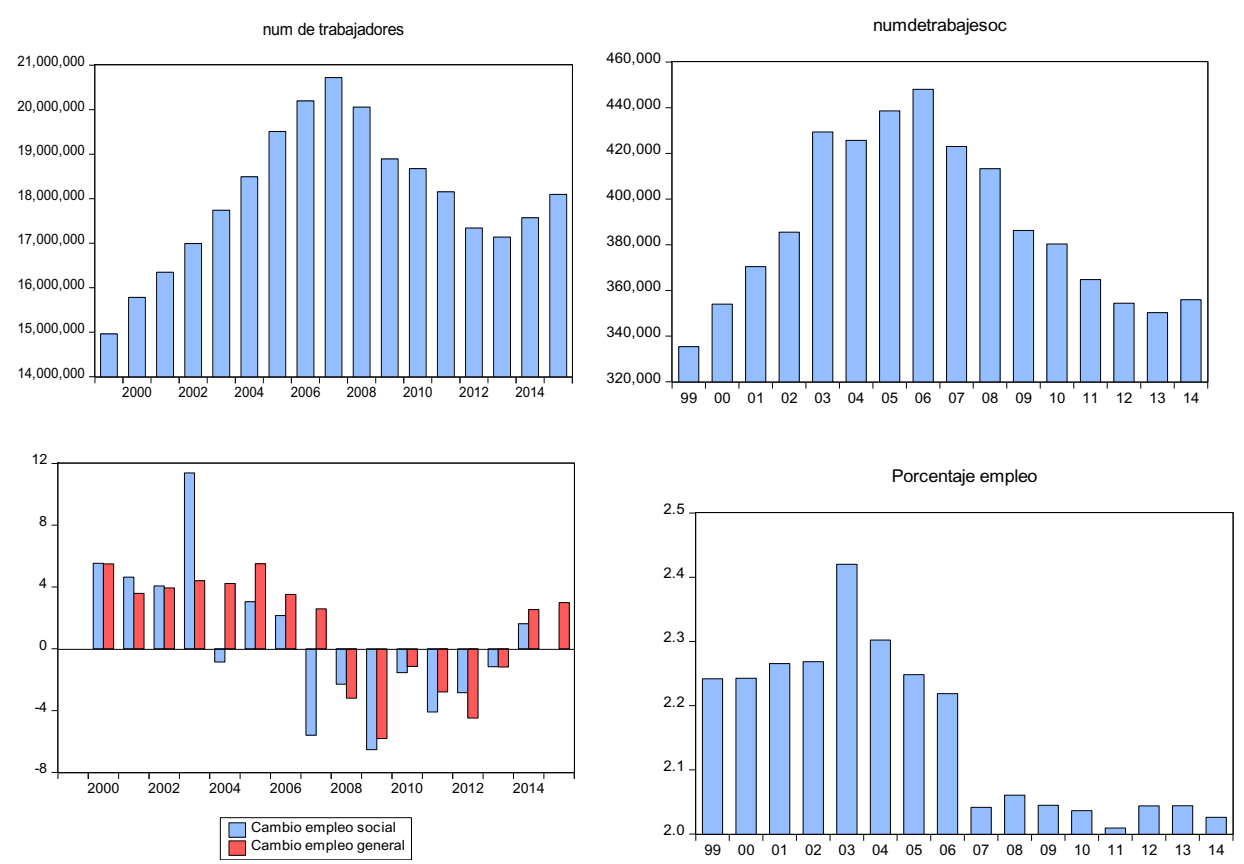

En la economía social se tiene un número máximo de trabajadores en 2006 aunque hay oscilaciones en años posteriores. El valor mínimo se tiene en 2013 con una ligera recuperación en 2014. En la economía general el máximo se alcanza en 2007 a partir del cual se empieza a descender, aunque con una ligera recuperación en 2014.

En términos porcentuales, la disminución entre el número máximo y mínimo de trabajadores es de un $16,38 \%$ en la economía social y un $15,9 \%$ en la economía en general. Estas variaciones son diferentes según sea el sector que consideremos. En la economía social, el más dañado es el sector de la construcción que ha bajado un $71,3 \%$ entre 2006 y 2014 mientras que el sector de los servicios se ha mantenido prácticamente constante, la agricultura ha bajado un $26,2 \%$ y la industria un $24,5 \%$. En el caso general el sector más dañado es, asimismo, la construcción con una 
bajada del $66,6 \%$ seguido de la industria con un $27,9 \%$ y los servicios y agricultura con un $4,6 \%{ }^{5}$.

Un análisis conjunto de la participación de la economía social en el valor añadido, empleo y número de empresas nos muestra claramente un rasgo no por conocido menos interesante de la economía social. Este sector es claramente creador de empleo y, en menor medida, de empresas. Mientras su contribución máxima al valor añadido total es del 1,21\% en 2007, la contribución al empleo y al número de empresas es siempre superior. En el primer caso más del doble y en el segundo un $35 \%$ o más. Muestra lo anterior un aserto conocido en economía social de que, incluso en situaciones de recesión, las empresas de economía social soportan el empleo y la creación de empresas pues generan un empleo más notable y fortalecen la cohesión económica. Sin embargo, la segunda parte de la afirmación en cuanto a que dichas empresas muestran un comportamiento anticíclico, de acuerdo con los resultados, no parece ser adecuada en la actual crisis aunque sí lo haya sido en crisis anteriores.

Gráfico. 5.
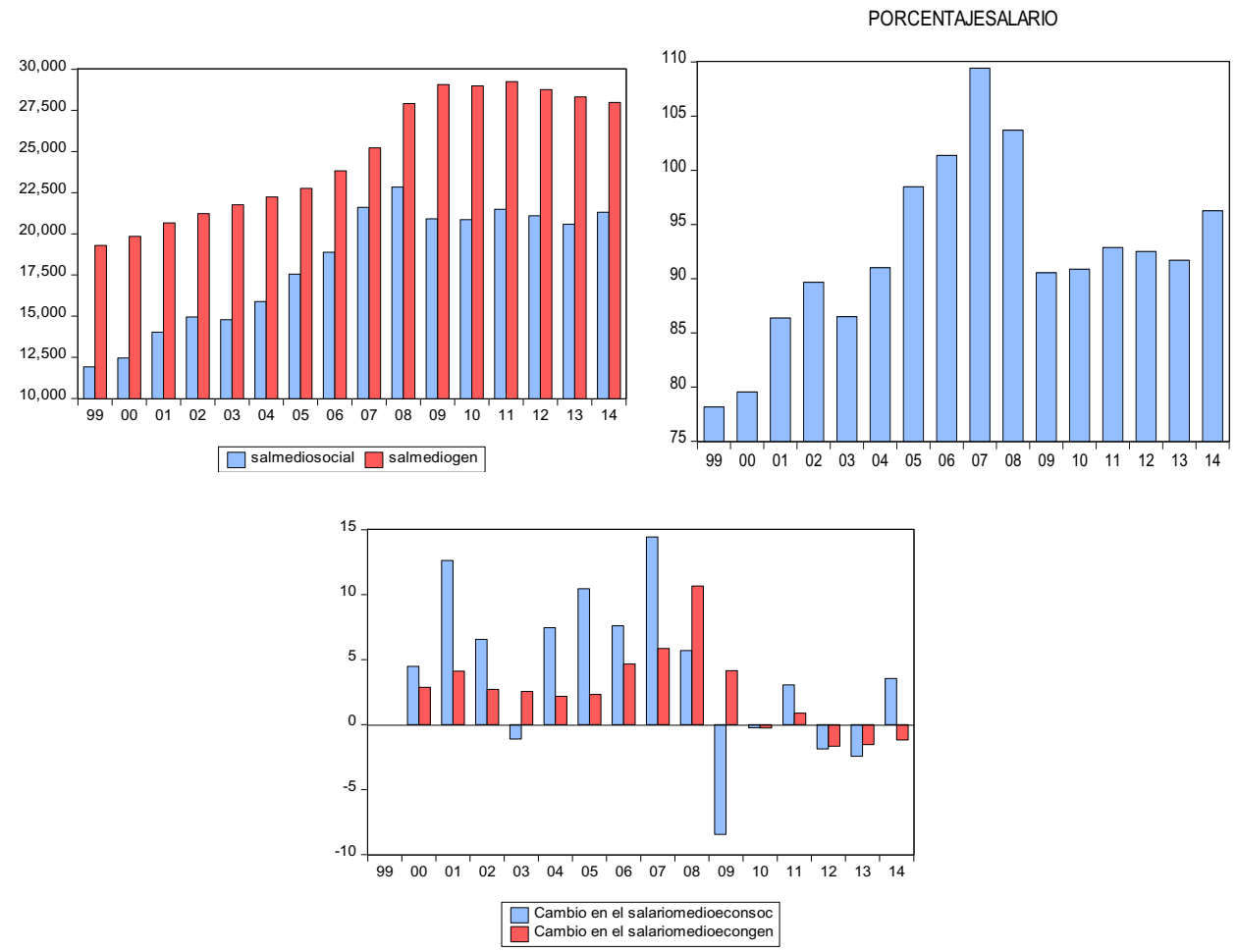

5 Los datos sectoriales están tomados de la Contabilidad Nacional (Empleo agregado por rama de actividad) (INE) y de la Dirección General de la Economía Social, del Trabajo Autónomo y de la Responsabilidad Social de las Empresas (Situación de empresas de Economía Social y sus trabajadores). 
El gráfico 5 muestra la evolución de los salarios medios en ambas economías. En la economía en general tiene su valor máximo en 2009 y a partir de ahí decrece ligeramente acorde con la disminución en los beneficios y el número de empleados. También hay que tener en cuenta que el salario de los directivos en la economía general suele ser mayor que en la economía social. No obstante, ha habido un retroceso que no se había recuperado en 2014. En cuanto a la economía social, el máximo se produce en 2008 aunque se ha producido una recuperación en los últimos años.

En términos de cambio anual, los descensos en la economía social han sido ligeramente mayores que en la economía general, aunque se ha producido un repunte en 2014.

Hay que tener en cuenta que, en forma general y en mayor medida en períodos recesivos, el salario en las empresas de economía social suele ser menor que en la economía en general dado que un objetivo de la economía social es la creación de empleo estable para sus socios y trabajadores en general, lo que puede suponer un menor salario aunque también hay que tener en cuenta, en el caso de los sociostrabajadores, la participación en los beneficios que puede traer consigo un complemento con respecto al salario.

En los gráficos 6 y 7 consideramos los beneficios medios de ambos tipos de empresas. En el gráfico 6 en relación al número total de empresas y en el 7 en relación al número total de empleados.

\section{Gráfico. 6.}
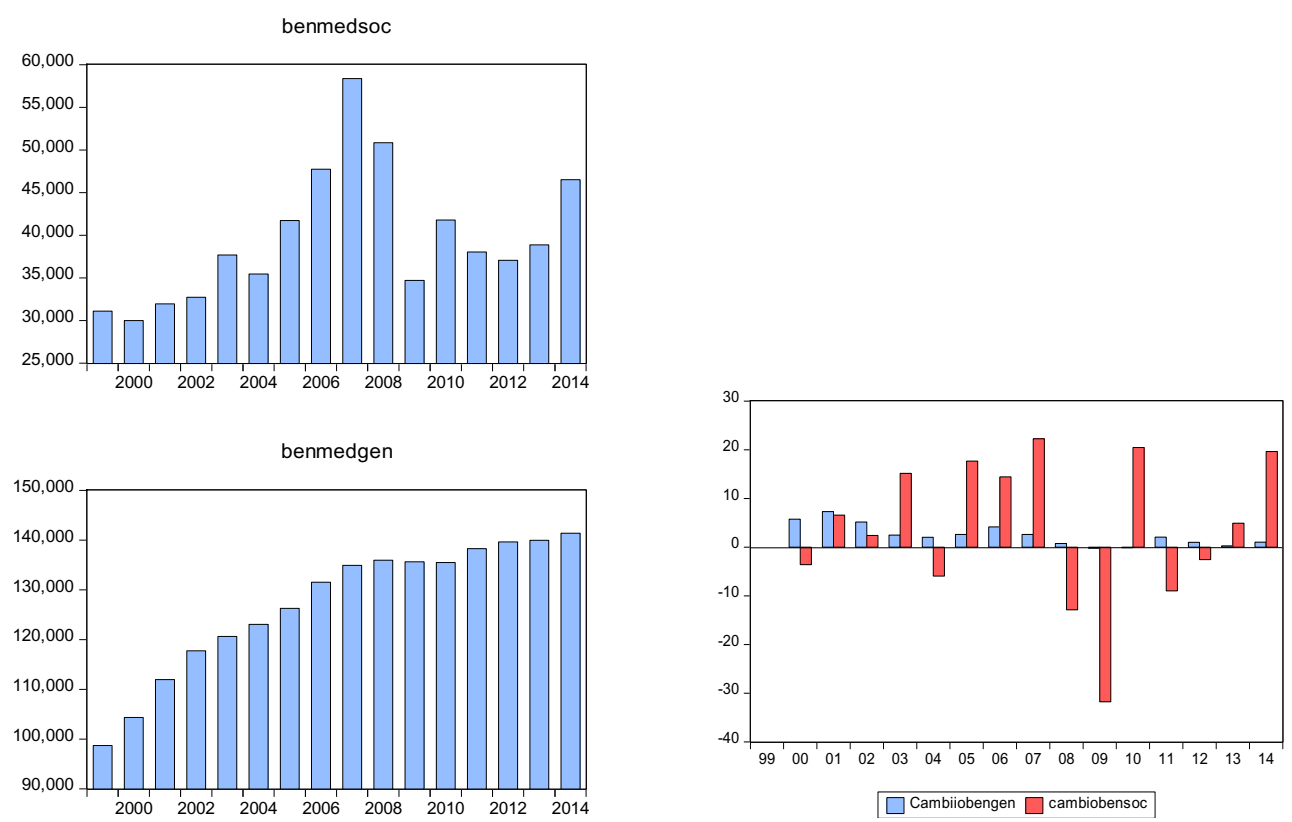

El gráfico 6 muestra, en principio, que los beneficios por empresa son mucho más bajos en la economía social que en la economía general. Probablemente ello 
sea debido a la pequeña dimensión de las primeras y a su poca expansión fuera de su ciudad o provincia mientras que la gran mayoría de las empresas medianas y grandes operan a escala nacional e incluso internacional. En segundo lugar, el objeto principal de las empresas de economía social no es el beneficio en sí mismo sino el bienestar de sus trabajadores. De hecho, el nivel de rentabilidad de las empresas de economía social es sensiblemente inferior al de las empresas lucrativas que operan en los mismos sectores de la actividad económica. La explicación viene dada por el hecho de que la actividad de cualquier empresa, sea capitalista o de economía social puede modelizarse como una producción conjunta de diversos bienes económicos de los que obtienen su renta los propietarios de los diversos factores productivos. Cualquier empresa produce dos tipos de renta, beneficios para el capital y salarios para el trabajador, dado que los beneficios de capital son mayores cuanto mayor es la flexibilidad con la que la empresa puede utilizar el trabajo, se deduce que la empresa de economía social es menos flexible que la capitalista pura y, por tanto, menos rentable. Se produce, por tanto, un intercambio entre la posibilidad de obtener un mayor salario o beneficio y la estabilidad en el empleo. Esto constituye una transferencia en especie que realizan las empresas de economía social en favor de sus empleados.

El primer gráfico muestra como 2007 es el año de mayor beneficio medio en la economía social, que además tiene fuertes altibajos con bajadas continuas en los beneficios de un año al siguiente. Los años de mayores bajadas de beneficios son 2009 y 2010 seguidos de 2011, 2012 y 2013 mientras que en las empresas en general sólo hubo pequeñas disminuciones en 2009 y 2010, manteniéndose o incluso aumentando el nivel de beneficios en las restantes.

Gráfico. 7.
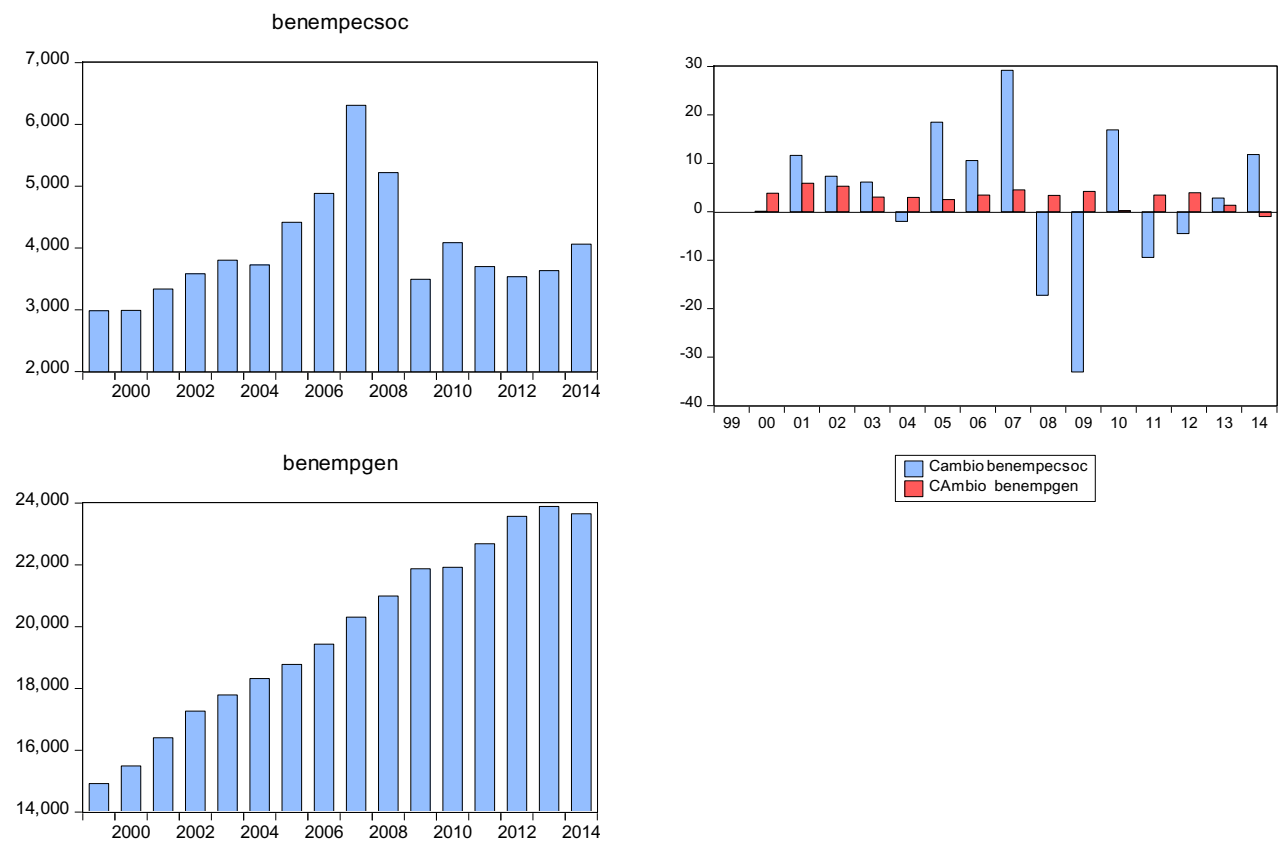

$\square$ Cambio benempecsoc 
El gráfico 7 muestra que los beneficios por empleado son bastante mayores en las empresas de la economía general que en las empresas de la economía social. Además la pauta con respecto a los años de crecimiento negativo se mantiene en ambos casos en relación a los beneficios medios. En consecuencia, este gráfico no añade ninguna reflexión adicional al anterior.

En el gráfico 8 se compara la evolución del empleo en la economía social y el PIB en el período 1999-2014.

Gráfico. 8.

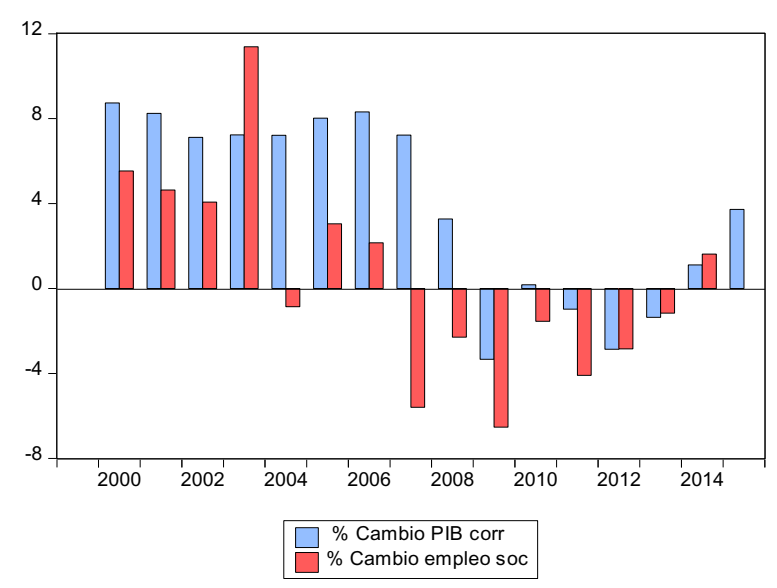

Se observa como en el período entre 1999 y 2006 ambos evolucionan de forma positiva en el mismo sentido de crecimiento, excepto en 2004, aunque el crecimiento en el empleo en la economía social es menor que en el PIB. Sin embargo, cuando entramos en el período recesivo, a partir de 2007, aunque el PIB tenga crecimientos muy pequeños el empleo en la economía social disminuye drásticamente, aunque en el año 2014 se comporta mejor, en términos de crecimiento, que el PIB. Podemos inferir, a partir de estos datos, que el comportamiento de la economía social es procíclico pues a una evolución positiva en el PIB corresponde una evolución positiva en el empleo y a un período de estancamiento o recesión corresponde un periodo de disminución de empleo social.

\section{Conclusiones}

En los apartados anteriores se ha intentado, de forma muy sencilla, comparar la evolución de la economía general con la economía social utilizando algunos indicadores. Tanto en cuanto a empleo como a número de empresas y el resto de las variables estudiadas, el comportamiento de la economía social es análogo que al de la general aunque nuestra hipótesis inicial fuese esperar un mejor comportamiento ante la crisis de la economía social debido a que esta última es 
más intensiva en trabajo que la general, aunque ello implique una menor productividad, y no está tan centrada en el beneficio como aquélla lo que implica una mayor rigidez en cuanto a la disminución de empleo y de los salarios. En conclusión, en una situación de crisis y de la salida de la misma parece que la economía social no tiene un mejor funcionamiento que la economía general. 


\section{ANEXO DE CUADROS}

Cuadro. 1. Datos de número de empresas, empleo y beneficio correspondientes a empresas de economía social

\begin{tabular}{|c|c|c|c|c|c|c|c|c|}
\hline Año & Empresas & $\mathrm{VAB}$ & Beneficios & $\begin{array}{l}\text { Beneficio } \\
\text { medio }\end{array}$ & Empleo & $\begin{array}{l}\text { Gasto en } \\
\text { personal }\end{array}$ & $\begin{array}{l}\text { Gasto } \\
\text { medio en } \\
\text { personal }\end{array}$ & $\begin{array}{l}\text { Beneficio } \\
\text { por } \\
\text { empleado }\end{array}$ \\
\hline 1999 & 32181 & 4998525,19 & 1000917,14 & 31102,73 & 335363 & 3997608,05 & 11920,24 & 2984,57 \\
\hline 2000 & 35269 & 5465597,31 & 1057605,6 & 29986,83 & 353933 & 4407991,7 & 12454,31 & 2988,15 \\
\hline 2001 & 38669 & 6431227,26 & 1235618,09 & 31953,71 & 370364 & 5195609,17 & 14028,38 & 3336,22 \\
\hline 2002 & 42191 & 7142760,51 & 1380452,53 & 32719,12 & 385450 & 5762307,98 & 14949,56 & 3581,40 \\
\hline 2003 & 43314 & 7979335,61 & 1632084,17 & 37680,29 & 429351 & 6347251,43 & 14783,36 & 3801,28 \\
\hline 2004 & 44747 & 8348219,86 & 1586069,05 & 35445,26 & 425660 & 6762150,81 & 15886,27 & 3726,14 \\
\hline 2005 & 46425 & 9633326,63 & 1936352,89 & 41709,27 & 438618 & 7696975,74 & 17548,24 & 4414,66 \\
\hline 2006 & 45821 & 10647472,8 & 2187237,86 & 47734,39 & 448046 & 8460234,9 & 18882,51 & 4881,72 \\
\hline 2007 & 45706 & 11817661,43 & 2667601,47 & 58364,3607 & 422968 & 9140059,97 & 21609,34 & 6306,86 \\
\hline 2008 & 42429 & 11596057,37 & 2157106,33 & 50840,37 & 413253 & 9438951,04 & 22840,61 & 5219,82 \\
\hline 2009 & 38898 & 9685496,45 & 1349290,33 & 34687,91 & 386254 & 8077013,47 & 20911,14 & 3493,27 \\
\hline 2010 & 37169 & 9485369,25 & 1552968,48 & 41781,28 & 380286 & 7932400,77 & 20859,03 & 4083,68 \\
\hline 2011 & 35487 & 9190074,00 & 1349290,33 & 38022,10 & 364736 & 7840783,67 & 21497,14 & 3699,36 \\
\hline 2012 & 33793 & 8727348,55 & 1251972,75 & 37048,28 & 354357 & 7475375,8 & 21095,60 & 3533,08 \\
\hline 2013 & 32734 & 8481150,01 & 1272368,18 & 38869,92 & 350243 & 7208781,83 & 20582,22 & 3632,81 \\
\hline 2014 & 31085 & 9031572,00 & 1445732,73 & 46509,01 & 355930 & 7585839,26 & 21312,72 & 4061,84 \\
\hline
\end{tabular}

Fuente: Dirección General de la Economía Social, del Trabajo Autónomo y de la Responsabilidad Social de las Empresas y elaboración propia 
Cuadro. 2. Evolución anual de los datos anteriores

\begin{tabular}{|l|l|l|l|l|l|l|l|l|}
\hline Año & Empresas & VAB & Beneficios & $\begin{array}{l}\text { Beneficio } \\
\text { medio }\end{array}$ & Empleo & $\begin{array}{l}\text { Gasto en } \\
\text { personal }\end{array}$ & $\begin{array}{l}\text { Salario } \\
\text { medio }\end{array}$ & $\begin{array}{l}\text { Beneficio } \\
\text { medio por } \\
\text { empleado }\end{array}$ \\
\hline 1999 & & & & & & & & \\
\hline 2000 & 9,59 & 9,34 & 5,66 & $-3,58$ & 5,53 & 10,26 & 4,48 & 0,11 \\
\hline 2001 & 9,64 & 17,67 & 16,83 & 6,55 & 4,64 & 17,86 & 12,63 & 11,64 \\
\hline 2002 & 9,10 & 11,06 & 11,72 & 2,39 & 4,07 & 10,90 & 6,56 & 7,34 \\
\hline 2003 & 2,66 & 11,71 & 18,22 & 15,16 & 11,38 & 10,15 & $-1,11$ & 6,13 \\
\hline 2004 & 3,31 & 4,62 & $-2,81$ & $-5,93$ & $-0,85$ & 6,53 & 7,46 & $-1,97$ \\
\hline 2005 & 3,75 & 15,39 & 22,08 & 17,67 & 3,04 & 13,82 & 10,46 & 18,47 \\
\hline 2006 & $-1,30$ & 10,53 & 12,95 & 14,44 & 2,14 & 9,91 & 7,60 & 10,57 \\
\hline 2007 & $-0,25$ & 10,99 & 21,96 & 22,26 & $-5,59$ & 8,03 & 14,44 & 29,19 \\
\hline 2008 & $-7,16$ & $-1,87$ & $-19,13$ & $-12,89$ & $-2,29$ & 3,27 & 5,69 & $-17,23$ \\
\hline 2009 & $-8,32$ & $-16,47$ & $-37,44$ & $-31,77$ & $-6,53$ & $-14,42$ & $-8,44$ & $-33,07$ \\
\hline 2010 & $-4,44$ & $-2,06$ & 15,09 & 20,44 & $-1,54$ & $-1,79$ & $-0,24$ & 16,90 \\
\hline 2011 & $-4,52$ & $-3,11$ & $-13,11$ & $-8,99$ & $-4,08$ & $-1,15$ & 3,05 & $-9,41$ \\
\hline 2012 & $-4,77$ & $.5,03$ & $-7,21$ & $-2,56$ & $-2,84$ & $-4,66$ & $-1,86$ & $-4,49$ \\
\hline 2013 & $-3,13$ & $-2,82$ & 1,62 & 4,91 & $-1,16$ & $-3,56$ & $-2,43$ & 2,82 \\
\hline 2014 & $-5,03$ & 6,49 & 13,62 & 19,65 & 1,62 & 5,23 & 3,54 & 11,81 \\
\hline & & & & & & & & \\
\hline
\end{tabular}

Fuente: Dirección General de la Economía Social, del Trabajo Autónomo y de la Responsabilidad Social de las Empresas y elaboración propia 
Cuadro. 3. Datos del número de empresas, empleo y beneficio correspondientes al total de empresas de la economía española.

\begin{tabular}{|l|l|l|l|l|l|l|l|l|}
\hline Año & Empresas & VAB & Beneficios & $\begin{array}{l}\text { Beneficio } \\
\text { medio }\end{array}$ & Empleo & $\begin{array}{l}\text { Gastos en } \\
\text { personal }\end{array}$ & $\begin{array}{l}\text { Salario } \\
\text { medio }\end{array}$ & $\begin{array}{l}\text { Beneficio } \\
\text { por } \\
\text { empleado }\end{array}$ \\
\hline 1999 & 2518801 & 538938 & 248589 & 124356,67 & 16664,9 & 228.162 & 13691,17 & 16617,13 \\
\hline 2000 & 2595392 & 586321 & 270834 & 135417 & 17485,2 & 247.113 & 15657,54 & 17160,61 \\
\hline 2001 & 2645317 & 636824 & 296172 & 148011,99 & 18058,9 & 265.514 & 16241,10 & 18116,47 \\
\hline 2002 & 2710400 & 682380 & 319075 & 159378,12 & 18482,1 & 283.305 & 16672,88 & 18778,06 \\
\hline 2003 & 2813159 & 727685 & 339380 & 169435,84 & 19080,2 & 303.208 & 17091,23 & 19130,23 \\
\hline 2004 & 2942583 & 776193 & 362133 & 180705,08 & 19769,6 & 322.788 & 17456,62 & 19584,49 \\
\hline 2005 & 3064129 & 834247 & 386878 & 192956,60 & 20600,9 & 347.684 & 17821,48 & 19830,54 \\
\hline 2006 & 3174393 & 900092 & 417480 & 208115,65 & 21486,7 & 376.212 & 18628,54 & 20672,03 \\
\hline 2007 & 3336657 & 972855 & 450170 & 224299,95 & 22170,8 & 409.174 & 19749,73 & 21728,55 \\
\hline 2008 & 3422239 & 1025672 & 465182 & 231664,34 & 22161,6 & 441.724 & 22025,25 & 23194,96 \\
\hline 2009 & 3355830 & 1006122 & 455174 & 226567,44 & 20811,4 & 436.308 & 23096,75 & 24095,51 \\
\hline 2010 & 3291263 & 989913 & 445879 & 221830,34 & 20338,8 & 428.708 & 22956,32 & 23875,84 \\
\hline 2011 & 3250576 & 983721 & 449397 & 223469,41 & 19815,2 & 420.210 & 23148,18 & 24756,07 \\
\hline 2012 & 3199617 & 954026 & 446722 & 222028,82 & 18953,7 & 395.378 & 22802,23 & 25763,40 \\
\hline 2013 & 3146570 & 935655 & 440393 & 218774,46 & 18434,9 & 384.592 & 22444,50 & 25701,07 \\
\hline 2014 & 3119310 & 943779 & 441025 & 218979,64 & 18645,7 & 388.993 & 22140,69 & 25102,31 \\
\hline 2015 & 3186878 & 975795 & 453015 & 224821,34 & 19153,6 & 404.208 & 22339,03 & 25036,47 \\
\hline
\end{tabular}

Fuente: INE y elaboración propia 
Cuadro. 4. Evolución anual de los datos anteriores

\begin{tabular}{|l|l|l|l|l|l|l|l|l|}
\hline Año & Empresas & VAB & Beneficios & $\begin{array}{l}\text { Beneficio } \\
\text { medio }\end{array}$ & Empleo & $\begin{array}{l}\text { Gastos en } \\
\text { personal }\end{array}$ & $\begin{array}{l}\text { Salario } \\
\text { medio }\end{array}$ & $\begin{array}{l}\text { Beneficio } \\
\text { por } \\
\text { empleado }\end{array}$ \\
\hline 1999 & & & & & & & & \\
\hline 2000 & 3,04 & 8,79 & 8,94 & 8,89 & 5,73 & 8,30 & 14,36 & 3,27 \\
\hline 2001 & 1,92 & 8,61 & 9,35 & 9,30 & 7,29 & 7,44 & 3,72 & 5,57 \\
\hline 2002 & 2,46 & 7,15 & 7,73 & 7,67 & 5,14 & 6,70 & 2,65 & 3,651 \\
\hline 2003 & 3,79 & 6,63 & 6,36 & 6,31 & 2,47 & 7,02 & 2,51 & 1,87 \\
\hline 2004 & 4,60 & 6,66 & 6,70 & 6,65 & 2,01 & 6,45 & 2,14 & 2,37 \\
\hline 2005 & 4,13 & 7,47 & 6,83 & 6,77 & 2,59 & 7,71 & 2,09 & 1,25 \\
\hline 2006 & 3,59 & 7,89 & 7,90 & 7,85 & 4,16 & 8,20 & 4,53 & 4,24 \\
\hline 2007 & 5,11 & 8,08 & 7,83 & 7,77 & 2,58 & 8,76 & 6,02 & 5,11 \\
\hline 2008 & 2,56 & 5,42 & 3,33 & 3,28 & 0,75 & 7,95 & 11,52 & 6,75 \\
\hline 2009 & $-1,94$ & $-1,90$ & $-2,15$ & $-2,20$ & $-0,21$ & $-1,22$ & 4,86 & 3,88 \\
\hline 2010 & $-1,92$ & $-1,61$ & $-2,04$ & $-2,09$ & $-0,12$ & $-1,74$ & $-0,61$ & $-0,91$ \\
\hline 2011 & $-1,23$ & $-0,62$ & 0,78 & 0,73 & 2,05 & $-1,98$ & 0,83 & 3,68 \\
\hline 2012 & $-1,56$ & $-3,01$ & $-0,59$ & $-0,64$ & 0,98 & $-5,91$ & $-1,49$ & 4,07 \\
\hline 2013 & $-1,65$ & $-1,92$ & $-1,41$ & $-1,46$ & 0,24 & $-2,73$ & $-1,57$ & $-0,24$ \\
\hline 2014 & $-0,86$ & 0,86 & 0,14 & 0,09 & 1,01 & 1,14 & $-1,35$ & $-2,33$ \\
\hline 2015 & 2,16 & 3,39 & 2,71 & 2,66 & 0,54 & 3,91 & 0,89 & $-0,26$ \\
\hline
\end{tabular}

Fuente: INE y elaboración propia 
Cuadro 5. Comparación entre diferentes magnitudes

\begin{tabular}{|c|c|c|c|}
\hline Año & $\begin{array}{l}\text { VAB Social sobre } \\
\text { el total }\end{array}$ & $\begin{array}{l}\text { Empleo social sobre el } \\
\text { total }\end{array}$ & $\begin{array}{l}\text { Empresas sociales } \\
\text { sobre el total }\end{array}$ \\
\hline 1999 & 0.93 & 2.24 & 1.28 \\
\hline 2000 & 0.93 & 2.24 & 1.36 \\
\hline 2001 & 1.01 & 2.26 & 1.46 \\
\hline 2002 & 1.04 & 2.26 & 1.55 \\
\hline 2003 & 1.09 & 2.42 & 1.54 \\
\hline 2004 & 1.07 & 2.30 & 1.52 \\
\hline 2005 & 1.15 & 2.24 & 1.51 \\
\hline 2006 & 1.18 & 2.22 & 1.44 \\
\hline 2007 & 1.21 & 2.04 & 1.37 \\
\hline 2008 & 1.13 & 2.06 & 1.24 \\
\hline 2009 & 0.96 & 2.04 & 1.16 \\
\hline 2010 & 0.96 & 2.03 & 1.13 \\
\hline 2011 & 0.93 & 2.01 & 1.05 \\
\hline 2012 & 0.91 & 2.04 & 1.04 \\
\hline 2013 & 0.90 & 2.05 & 0.99 \\
\hline 2014 & 0.95 & 2.02 & \\
\hline
\end{tabular}

Fuente: INE, Dirección General de la Economía Social, del Trabajo Autónomo y de la Responsabilidad Social de las Empresas y elaboración propia

\section{Referencias bibliográficas}

Algora, J. M. (2011) La economía social: Crisis y recuperación económica. Haol. № 25, pp $133-140$.

Alianza Cooperativa Internacional (1995) Asamblea General. Manchester.

Bacchiega, A. y Borgaza, C. (2003) The economics of the third sector: A more comprehensive approach. En H.K. Anheier y A. Ben Ner (eds), The study of non profit enterprises: Theories and approaches. Nueva York: Kluwer.

Bretos, I. y Morandeira, J. (2016) La economía social ante la actual crisis económica en la Comunidad Autónoma del País Vasco. REVESCO. Revista de Estudios Cooperativos, No 122, pp. 7-33. DOI: 10.5209/rev_REVE.2016.v122.52020.

Chaves, R. y Monzón, J.L. (2008) La Economía Social en la Unión Europea. Bruselas: Comité Económico y Social Europeo.

Dirección general de la economía social, del trabajo autónomo y de la responsabilidad social de las empresas (varios años): Datos sobre cooperativas y sociedades laborales. Página web: www.empleo.gob.es/es/organizacion/empleo.

Instituto Nacional de Estadística (INE) (varios años): Datos de la economía general. Página web www.ine.es

Pérez de Mendiguren, J. C., Etxezarreta, E. y Guridi, L. (2008) ¿De qué hablamos cuando hablamos de economía social y solidaria? Concepto y nociones afines. XI Jornadas de Economía Crítica.

Román, C. (2014) Las cooperativas españolas y los ciclos económicos. Un análisis comparado. Ciriec-España. Revista de Economía, Pública, Social y Cooperativa, № 80 pp.77-109. 
Saéz, F. y González, F. (2005) Literatura y realidad del tercer sector. Un panorama comparado en García Delgado, J. L. (dir): Un enfoque económico de la economía social. Fundación Once para la cooperación e integración social de las personas con discapacidad.

Sala, M., Farré, M y Torres, T. (2014) Un análisis del comportamiento cíclico de las cooperativas y sociedades laborales españolas y su relación con la actividad económica. REVESCO. Revista de Estudios Cooperativos, No 115, pp. 7-29. DOI: 10.5209/rev_REVE.2014.v115.45279. 